\title{
De la station au territoire : quels contours pour les destinations touristiques?
}

Hugues François et Sylvain Billet

\section{CpenEdition}

\section{Journals}

Édition électronique

URL : http://journals.openedition.org/tourisme/280

DOI : 10.4000/tourisme.280

ISSN : 2492-7503

Éditeur

Éditions touristiques européennes

Édition imprimée

Date de publication : 1 décembre 2010

Pagination : 87-98

ISSN : 2109-5671

Référence électronique

Hugues François et Sylvain Billet, « De la station au territoire : quels contours pour les destinations

touristiques? », Mondes du Tourisme [En ligne], 2 | 2010, mis en ligne le 30 septembre 2015, consulté le 02 mai 2019. URL : http://journals.openedition.org/tourisme/280 ; DOI : 10.4000/tourisme.280

\section{c) $(1) \odot$}

Mondes du tourisme est mis à disposition selon les termes de la licence Creative Commons Attribution - Pas d'Utilisation Commerciale - Pas de Modification 4.0 International. 


\section{De la station au territoire :}

\section{quels contours pour les destinations touristiques ?}

\author{
Hugues FrançOIS
} [hugues.francois@cemagref.fr]

Ingénieur de recherche, Cemagref Grenoble, Terriscope

Résumé. Dans le contexte du post-tourisme, les stations de sports d'hiver situées à proximité d'aires urbaines constituent un laboratoire d'observation de tendances plus générales. Dans le contexte de la périurbanisation, l'interpénétration des sphères du tourisme et du quotidien y est exacerbée. Fragmentation des séjours (réduits de un à trois jours, voire à de l'excursionisme à la journée) et nouvelles formes de résidentialité y sont souvent plus marquées. Ces dynamiques pèsent fortement sur l'évolution du modèle économique des stations fondé sur le séjour touristique. Dans cet article, nous proposons d'analyser ce phénomène au regard de l'ancrage territorial, traditionnellement considéré comme un facteur d'adaptation. À travers deux cas d'étude contrastés dans le pourtour de l'agglomération grenobloise, les Sept-Laux et Saint-Pierre-de-Chartreuse, nous montrerons comment cet ancrage peut également être une variable adaptative.
SYLVAIN B LLLET_[sbillet@terriscopefr]

Chargé de mission, Terriscope

Abstract. In the context of post-tourism, ski resorts located near urban areas are a laboratory observation of broader trends. In the context of suburbanization, the interpenetration of the spheres of tourism and daily life is exacerbated. Fragmentation of stay (reduced to one to three days, or even to day trippers) and new forms of daily migration are often more marked there. These dynamics strongly influence the evolution of the economic model of the resorts based on the long stay holidays. In this paper, we propose to analyze this phenomenon in terms of local anchorage, traditionally regarded as an adaptation factor. Through two contrasted case studies in the periphery of Grenoble, Sept-Laux and Saint-Pierre-de-Chartreuse, we will show how this anchoring can also be an adaptive variable. 


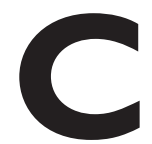

onçues au sortir de la deuxième guerre mondiale, les stations de sports d'hiver sont le fruit de l'affirmation du régime de régulation fordiste (Cuvelier, 1997) : elles s'inscrivent dans le développement des loisirs “compensatoires”, d'une part, et elles sont conçues selon la rationalité de l'intégration fonctionnelle, de l'autre. Si ce modèle est a priori a-territorial, son ancrage repose avant tout sur les effets d'entraînement d'un pôle de croissance. Le terme "d'industrie de la neige" est souvent associé à la lourdeur des équipements de transport (i.e. les remontées mécaniques) et la filière associée. Cette expression contribue directement à la mise en œuvre du Plan neige qui, en l'absence de planification réelle, constitue avant tout une doctrine d'aménagement touristique de la montagne. Le Plan neige désigne alors un modèle de conception de la station dite intégrée, en site vierge, ex nihilo ou de troisième génération, cette dernière dénomination étant particulièrement révélatrice de l'intervention normative de l'État (François, 2007).

Aujourd'hui, le concept même de station est remis en cause par l'évolution des pratiques, non seulement touristiques, mais également par le développement de nouvelles formes de "résidentialité". Fragmentation des séjours et développement des courts séjours, voire de l'excursionnisme (Lazzarotti, 1994 ; Knafou et Violier, 2005 ; Lazzarotti,
2005), et transformation des résidences touristiques en résidences principales (Briquel, 200 I ; Davezies et Lejoux, 2003; Bryant, 2005) sont autant de facteurs qui mettent à mal le modèle de tourisme de séjour sous-tendu par l'intégration fonctionnelle. En effet, au-delà de l'intégration, le déploiement parallèle de l'immobilier de loisirs et du domaine skiable induit un développement économique fondé sur le séjour touristique et sur les dépenses d'une clientèle captive dans un seul et unique lieu. Ainsi, en moyenne montagne, où les concepts de l'intégration fonctionnelle n'ont pu être respectés à la lettre, la logique du séjour demeure.

Or ces stations de moyenne montagne sont plus directement frappées par les “perturbations” évoquées précédemment. Plus accessibles que leurs concurrentes d'altitude, elles se prêtent donc mieux à une fréquentation limitée dans le temps et, par ailleurs, elles sont plus attractives du point de vue de l'immobilier résidentiel. Dans ces stations, on assiste alors à une forme de déterritorialisation, ainsi qu'à une prise d'autonomie de la part du gestionnaire de l'entreprise centrale de remontées mécaniques. Ce dernier se concentre sur sa propre rentabilité et optimise l'utilisation de son appareil productif en fonction de critères internes et sans tenir compte des rythmes qui animent la vie du territoire. Nous pouvons par ailleurs constater que le fonctionnement de la station ne repose plus sur sa seule "commune support", mais trouve une cohérence dans un territoire plus large, par exemple celui d'un parc naturel régional. En particulier, le logement touristique n'est plus nécessairement interne à la station, le ski devient alors une activité parmi d'autres au sein d'une offre de territoire.

Dès lors, l'ancrage territorial tend à changer de nature et la station voit son statut et son rôle évoluer vis-à-vis de son territoire support : si le tourisme hivernal demeure une activité économique importante par son volume, elle n'est plus nécessairement la locomotive du développement ; l'attractivité de la station dépend en grande partie de son écrin local (François, 2007). L'objet de cet article ${ }^{(1)}$ est donc d'expliciter comment le concept de la station, conçue pour fixer les touristes au sein d'une destination bien délimitée, est mis à mal et comment il s'intègre désormais à des flux pertinents au regard des pratiques de loisirs actuelles.

\section{DES STATIONS SOUS INFLUENCE URBAINE}

Les stations situées en périphérie d'agglomération subissent de plein fouet les deux tendances évoquées plus haut, à savoir la résidentialisation et la fragmentation des séjours touristiques. Dans ce sens, nous les considérons comme des cas extrêmes de leur concrétisation et donc comme des laboratoires pour mieux comprendre l'impact 
de ces phénomènes sur les trajectoires de développement des stations. Celles qui retiendront plus particulièrement notre attention dans cette première partie sont la station village Saint-Pierre-deChartreuse et la station de troisième génération Les Sept-Laux, toutes deux situées dans un isochrone de 40 minutes depuis Grenoble (cf. figure 1).

Avant de nous focaliser sur ces cas d'études, nous reviendrons toutefois sur le contexte périurbain auquel sont confrontées ces stations. Ensuite, nous présenterons plus précisément un indicateur particulier des tendances à l'œuvre en nous appuyant sur leurs dynamiques résidentielles particulières, à l'aune des données démographiques disponibles.

\section{La dynamique périurbaine}

Le marché périurbain du tourisme constitue un objet de recherche méconnu. En effet, la littérature relativement abondante sur la périurbanisation ne s'intéresse pas directement à cette question, l'étude de la périurbanisation visant surtout à évaluer l'importance des mouvements entre l'agglomération et l'espace rural proche, et l'autonomie de ce dernier. Dans ce sens, la recherche observe essentiellement les migrations pendulaires en s'appuyant particulièrement sur les données de l'Insee.

D'autres approches recherchent des critères pertinents d'évaluation de la périurbanisation, comme celle de Vincent Briquel (200I) fondée sur les zones de chalandise des communes et sur les formes d'intercommunalité auxquelles elles participent. Si elles apportent des informations contextuelles, ces données ne permettent pas spécifiquement de parler de la situation touristique. Une approche de la périurbanisation par le foncier semble a priori plus instructive (Guillaud-Lauzanne, 2004) mais montre rapidement ses limites, notamment parce que, en la matière, il est particulièrement difficile de distinguer les effets du tourisme de ceux de la périurbanisation. Cet entremêlement des deux phénomènes sera d'ailleurs au cœur de nos préoccupations.

L'étude de Guillaud-Lauzanne (2004) s'intéresse tout particulièrement au cas de la Chartreuse. Malgré une étude approfondie des pressions foncières sur plusieurs communes du massif, elle conclut que le cas de Saint-Pierre-deChartreuse est particulièrement difficile à interpréter tant les effets de périurbanisation et de tourisme se confondent, notamment du point de vue de l'urbanisme. Par exemple, quand elle observe la croissance

Figure I

Localisation de la zone d'étude:

\section{Saint-Pierre-de-Chartreuse et les Sept-Laux}

deux stations dans un isochrone de 35-40 minutes depuis Grenoble

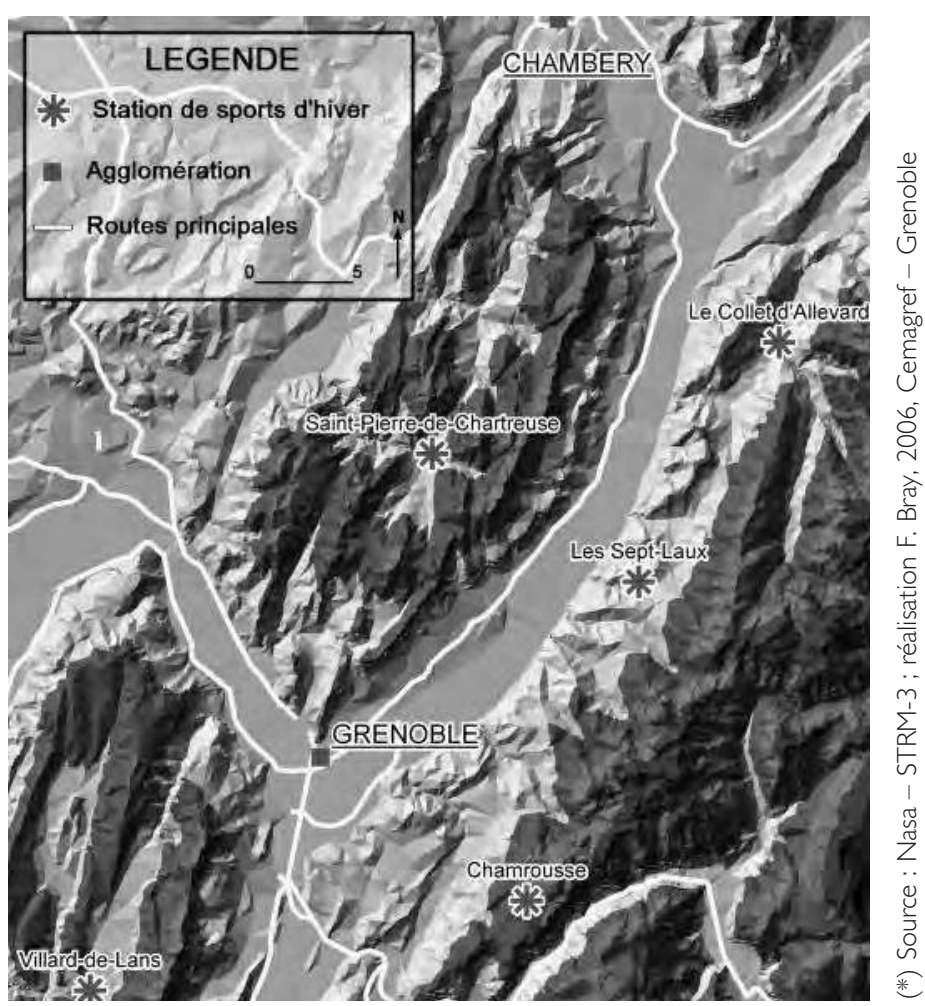


Figure 2

\section{Taux de croissance des populations communales}

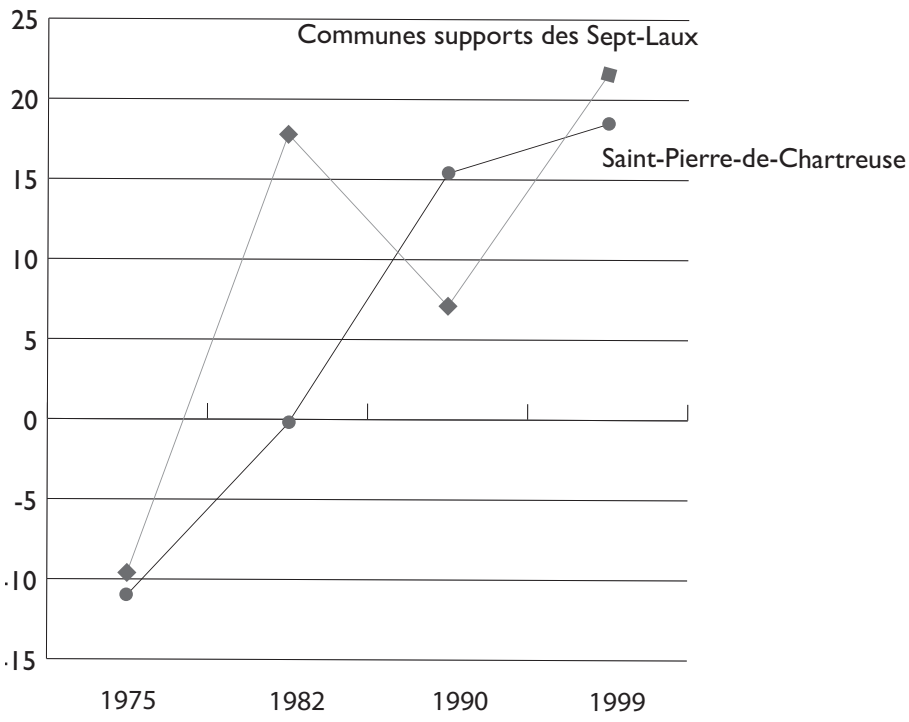

Figure 3

Part des migrations et de la natalité dans les mouvements de population

Pourcentage

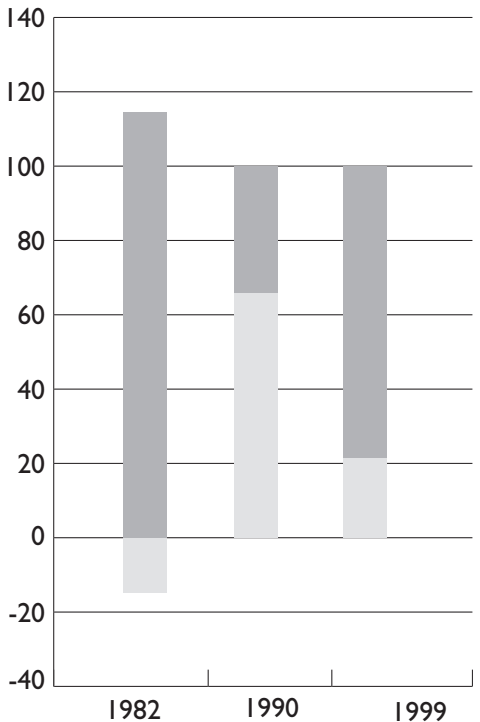

Part des migrations aux Sept-Laux

Part de la natalité aux Sept-Laux
Pourcentage

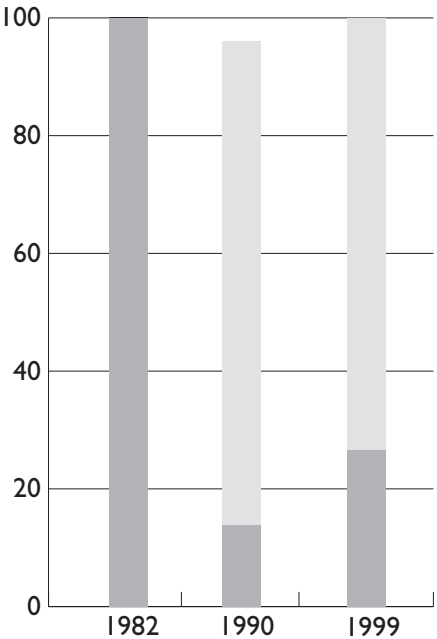

Part de la natalité à Saint-Pierre-de-C.
Part des migrations à Saint-Pierre-de-C. des notifications Safer à des fins de loisirs, elle constate que, "même si la commune de Saint-Pierre-deChartreuse voile probablement une partie de la réalité de cette observation par son activité touristique, il est avéré que les pratiques foncières périurbaines s'accompagnent fréquemment de pressions foncières originales (golfs, tennis...) encourageant la hausse des prix" et "les activités touristiques mises en valeur par le parc naturel régional de Chartreuse motivent sans conteste de telles destinations" à des fins résidentielles. Face à ces difficultés, nous proposons, tel que le présente Vincent Briquel (200I), d'analyser les tendances à la périurbanisation des différentes communes grâce aux données démographiques. Pour cet auteur, les communes qui nous intéressent sont classées comme suit : Les Adrets et Theys sont qualifiées de "strictement périurbaines", alors que La Ferrière et Saint-Pierrede-Chartreuse sont dites "non périurbaines". Afin de constater les différentes évolutions, nous proposons donc une vision des mouvements de population afin d'en estimer les tendances.

Comme la figure 2 permet de le constater, les communes passent, entre 1975 et 1982, d'une situation où la population tend à diminuer, à une situation où elle semble relativement stabilisée à Saint-Pierrede-Chartreuse et où elle commence déjà à augmenter aux Sept-Laux. Cependant, nous pouvons également observer que l'évolution démo- 
graphique suit des chemins légèrement différents si on se situe à SaintPierre-de-Chartreuse où elle ne cesse d'augmenter, alors que les communes supports de la station des Sept-Laux voient leur croissance ralentir entre 1982 et 1990.

Il est intéressant de mettre en parallèle ces observations avec l'importance relative que prennent les soldes naturels et migratoires dans ces mouvements de population (cf. figure 3).

En effet, l'augmentation importante du taux de croissance de la population entre les années 1975 et 1982 est surtout due à de fortes migrations, puisque le solde naturel est négatif. Par la suite, la baisse des années 1990 semble due à des migrations moins importantes qui n'expliquent plus que $34 \%$ des mouvements de population, mouvements qui redeviennent plus importants quand la part des migrations devient plus forte. Ainsi, il semblerait que l'installation de personnes étrangères à ces communes constitue le facteur le plus important de leur croissance démographique. Il en va de même à Saint-Pierre-deChartreuse qui, faute de migration dans un premier temps, a un taux de croissance de la population qui augmente mais reste tout de même négatif. Par la suite, on voit que la progression importante et continue de ce dernier repose essentiellement sur les migrations. De même, entre 1990 et 1999 , on peut constater que si les naissances prennent une place plus importante dans l'augmentation du nombre d'habitants, tout en restant minoritaires, le taux de croissance démographique tend à ralentir sa progression. Ainsi, nous pouvons conclure, a priori, que la périurbanisation à SaintPierre-de-Chartreuse est un phénomène plus récent mais qui tend aujourd'hui à s'affirmer.

Du point de vue des loisirs, la fréquentation périurbaine, également vérifiée par une enquête au pied des pistes ${ }^{(2)}$, indique une présence importante des clientèles de proximité comme le confirme la durée des séjours en station (cf. figure 4).

Néanmoins, la notion de fréquentation de proximité dépasse la seule agglomération grenobloise au sens strict et peut concerner d'autres centres d'émissions, parfois plus éloignés. En ce sens, les stations périurbaines font réellement figure de laboratoire des tendances actuelles. Pour les stations étudiées, cette réalité concerne plus particulièrement la clientèle lyonnaise, présente notamment le week-end.

La présence d'infrastructures liées à l'activité du centre urbain n'est sans doute pas sans rapport avec l'accessibilité de ces stations. Ce fait appelle l'observateur à extrapoler. Par exemple, la fréquentation par les clientèles originaires du bas-

Figure 4

Durée des séjours dans les stations de Saint-Pierre-de-Chartreuse et des Sept-Laux

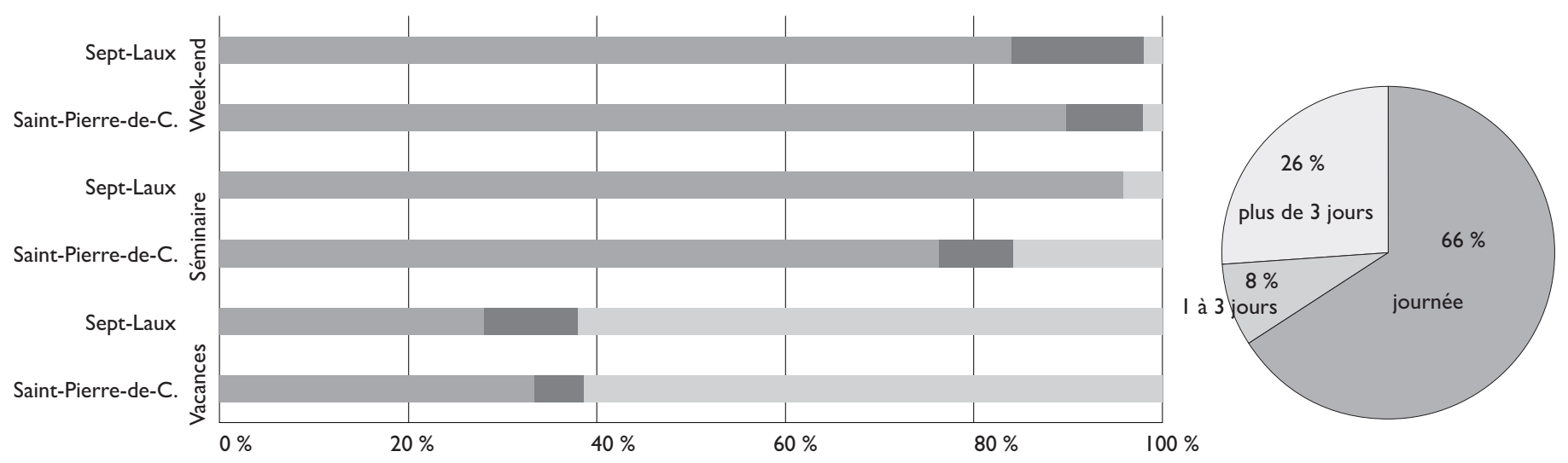


sin chambérien et annécien des stations de la Tarentaise est facilitée par un réseau de transport de qualité, pour partie organisé en fonction des besoins touristiques (favorisant l'accès aux stations et capable d'amortir partiellement les pics de fréquentation). En outre, ces centres urbains sont un facteur d'attractivité. Ainsi, un élément inattendu ressort de l'enquête auprès des clientèles citée précédemment : la présence de consommateurs qui ne résident pas directement en proximité des stations étudiées mais qui sont de passage dans l'agglomération pour des raisons professionnelles et qui profitent des stations proches pour une pratique de loisirs purs. Contrairement aux facteurs d'attractivité de la station (cf. infra), ici, c'est avant tout la renommée du domaine skiable qui joue en faveur de la station.

\section{L'attractivité résidentielle des territoires supports de station}

En sus de cette dynamique des loisirs, l'évolution du parc de résidences des stations constitue un témoin, ainsi qu'un facteur actif de l'impact de la périurbanisation. En effet, ce parc constitue une pierre d'achoppement fondamentale entre tourisme et périurbanisation : la diminution du nombre de lits touristiques constitue un problème majeur pour des stations construites sur un modèle de tourisme de séjour, alors que, dans le même temps, elle pousse la station à capter de nou- veaux revenus de l'agglomération. Dans cette optique, nous mobiliserons les données relatives aux résidences secondaires (souvent utilisées comme un indicateur du volume de l'activité touristique) et celles propres aux résidences principales, réservées à des fins d'habitat.

La figure 5 nous permet de faire plusieurs observations. Si le nombre de résidences secondaires par habitant est relativement proche dans les deux stations à la fin des années 1960 (avant la création de la Zac des Sept-Laux), on distingue très nettement des voies de développement différentes se dessiner. De manière globale, on peut constater que, dans un premier temps, la commune de Saint-Pierre-deChartreuse, forte de son passé touristique, présente un taux de résidences secondaires supérieur à celui des communes supports des SeptLaux. Dans un deuxième temps, les variations que connaît SaintPierre-de-Chartreuse sont moins fortes et se trouvent largement dépassées par l'évolution de la situation aux Sept-Laux. Ainsi, le "démarrage" des sports d'hiver entre les périodes censitaires de 1968 et 1975 provoque une légère augmentation dans chacun des cas. Puis, à grand renfort de programmes immobiliers, la station des SeptLaux "décolle" littéralement et se constitue un stock non négligeable de résidences secondaires. Celui-ci semble relativement figé par rapport à l'augmentation de la population, de sorte que le rapport résidence / habitant ne fait ensuite que diminuer. À Saint-Pierre, au contraire, l'évolution est plus lente avec une légère tendance à la baisse entre 1975 et 1982, alors que la pression périurbaine, qui se précise dans la période suivante, conduit à un programme de construction qui fait légèrement remonter le ratio mais qui n'enraye pas véritablement sa décroissance; au mieux elle permet d'y résister. En effet, les variations que nous venons de décrire s'appliquent également à la part des résidences secondaires par rapport au nombre de résidences principales (cf. figure 6).

Cependant, si la diminution paraît plus forte aux Sept-Laux qu'à Saint-Pierre-de-Chartreuse, ce phénomène doit être relativisé. En effet, il ne révèle pas nécessairement les mêmes réalités : il paraît assez improbable que les studios cabines construits aux Sept-Laux fassent l'objet d'une demande à des fins de résidence principale! Par ailleurs, les variations que connaît la situation chartroussine amènent à souligner l'importance des nouvelles constructions dans l'évolution de ce rapport. Son augmentation entre 1982 et 1990 correspond plus à une nouvelle tranche de travaux qu'à une évolution du parc immobilier existant. De même, la diminution du ratio aux Sept-Laux conduit sans doute plus à s'interroger sur les nouvelles constructions au sein des villages plutôt qu'à une substitution des utilisations des 
logements en station. La distinction fonctionnelle des espaces de loisirs et de résidence, dans le cas de la station de troisième génération, constitue effectivement un facteur déterminant des trajectoires de développement des stations étudiées. La réalisation d'entretiens complémentaires indique, en effet, que les résidents permanents de la station des Sept-Laux sont encore peu nombreux et que le logement occupé n'est pas pour autant déclaré comme résidence principale.

À Saint-Pierre-de-Chartreuse, la confusion entre la station et le village constitue une caractéristique centrale, et des informations complémentaires ont pu être recherchées dans les permis de construire délivrés par la commune. Si les muta- tions tourisme - résidentiel n'apparaissent pas clairement du fait de la structure des hébergements marchands au sein du village, l'étude des permis et autorisations de travaux nous montre néanmoins un phénomène intéressant. Le cœur de station, départ des pistes, apparaît relativement épargné par les nouvelles constructions, qui se concentrent sur les zones marginales. Dans ces secteurs, ces constructions contribuent fréquemment à la transformation du patrimoine délaissé par l'agriculture, hypothéquant par là même une voie de diversification possible des hébergements vers un tourisme valorisant son ancrage territorial, et limitant sans doute les potentialités futures de développement touristique.

\section{L'ANCRAGE TERRITORIAL COMME \\ VARIABLE D’AJUSTEMENT}

Un postulat largement répandu dans le corpus de l'économie territoriale consiste à considérer l'ancrage comme un facteur soutenant la capacité d'adaptation des SPL (systèmes productifs locaux), et, dans notre cas, des STL (systèmes touristiques locaux) (Perret, 1992). L'ancrage territorial s'entend ici comme l'intensité des relations, notamment informelles, entre acteurs économiques au sein d'un SPL donné et la participation des activités considérées, dans notre cas la station, à la dynamique du substrat territorial et à sa construction sociale et culturelle. L'étude des “global value
Figure 5

Taux de résidences secondaires par habitant
Figure 6

\section{Taux de résidences secondaires par résidence principale}
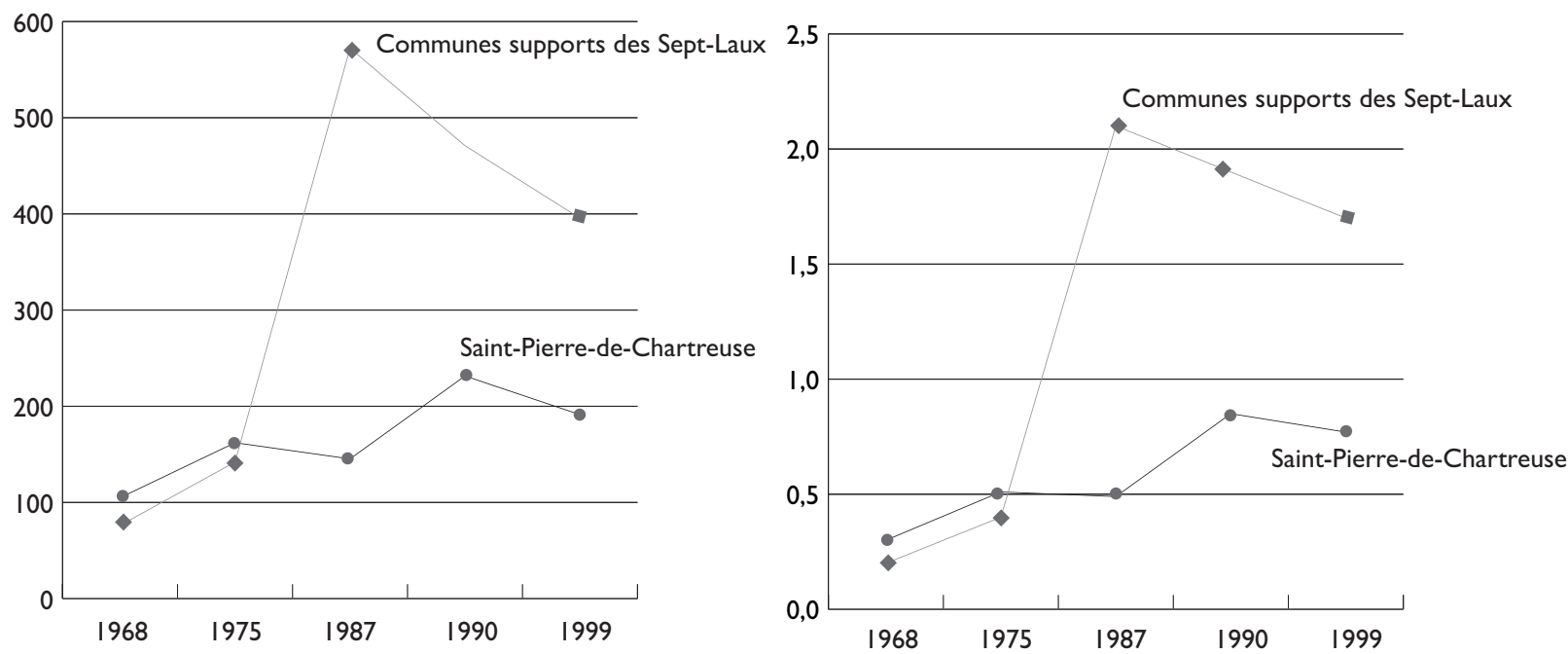
chains" (Schmitz et Nadvi, 1999) nous délivre par exemple l'image de clusters industriels s'inscrivant dans le mouvement de mondialisation grâce à leur flexibilité et à leur capacité de réponse à la demande ; insertion dans un réseau social dense, échanges de main-d'œuvre et de savoir-faire, faible taille des unités productives ou encore production par petits lots sont autant de facteurs constitutifs de la cohérence du système jouant en faveur de leur capacité d'adaptation.

Cependant, dans le cas des stations, la proximité urbaine bouleverse le modèle économique du séjour, brique de base de leur construction, et remet en cause la complémentarité des prestations touristiques constitutives de la destination. La cohérence productive et territoriale des stations s'en trouve ainsi bouleversée. L'ancrage, plus qu'un facteur d'adaptation, constitue alors une variable sur laquelle jouer (intensité et nature de l'ancrage) en réponse au problème posé. Ce processus apparaît plus particulièrement à travers l'évolution de l'offre de logement au regard des prestations de loisirs. Nous pouvons alors constater la mise en marche de processus de déterritorialisation et de reterritorialisation des stations, témoignages des profondes mutations de leur ancrage.

\section{Contraction de l'offre}

d'hébergement

L'immobilier de loisirs à Saint-

Pierre-de-Chartreuse. La structu- ration du tourisme sur le territoire de Saint-Pierre-de-Chartreuse s'inscrit pleinement dans la dynamique traditionnelle des stations villages qui considèrent le ski comme une locomotive pour le développement villageois. Le tourisme chartroussin trouve son origine dès le xixe siècle. Il s'agit alors d'un tourisme essentiellement religieux maîtrisé par les pères chartreux. Entre les deux guerres, le relais sera assuré par le Touring club de France et l'Automobile club de France, et ce n'est qu'au sortir de la Deuxième Guerre mondiale que les acteurs locaux vont réellement s'approprier l'activité touristique. Les espoirs placés dans le développement des sports d'hiver sont particulièrement forts et visent à retrouver un âge d'or que les habitants ont le sentiment de ne pas avoir saisi au moment opportun (Mauz et al., 1996). La mairie, ainsi que les ressources municipales en bois, sont ainsi mises à forte contribution : à elles d'équiper la station, les villageois prenant à leur charge le développement des services nécessaires à l'accueil des touristes. Si le modèle du Plan neige connaît ici une application tout à fait marginale, reste néanmoins le principe des effets d'entraînement. Ils contribuent même de manière originale à renforcer l'ancrage de la station, le fonctionnement des remontées mécaniques étant étroitement lié au dynamisme des initiatives locales.

L'immobilier de loisirs développé au sein du patrimoine local prend donc ici une forme particulière et relativement variée au regard des standards de la construction de studios cabines adoptés par les stations de troisième génération. La transformation d'usage de ce patrimoine conduit par ailleurs à un fort de taux de logements par habitant. Ces deux éléments concourent à l'attractivité résidentielle de la station, malgré son éloignement de l'agglomération grenobloise, amplifié par le passage parfois difficile du Col de Porte, qui a pendant longtemps contenu l'expansion périurbaine en direction de Saint-Pierre.

Si cette dynamique de confusion entre tourisme et résidence est parfois difficile à saisir (cf. supra, analyse du recensement général de la population), elle est soulignée par des faits saillants tels que la transformation d'un hôtel central et symbolique, l'hôtel du Parc, en studios destinés à une location annuelle. En sus de ces mouvements immobiliers, le partage des services entre résidents permanents et temporaires pose problème. Outre les questions traditionnelles de conflits d'usage, se pose ici celle de la capacité d'accueillir le public touristique traditionnel de la station, notamment les familles. La gestion de la crèche par les nouveaux habitants, surdimensionnée à des fins d'accueil touristique, a pu conduire à une fermeture de l'établissement durant les vacances de février, période de pointe pour la fréquentation touristique.

En complément de ces exemples 
de collision frontale des dynamiques résidentielle et touristique, des phénomènes originaux émergent. À ce titre, le rachat de résidences secondaires par des personnes habitant à proximité de la station, parfois dans les communes membres du PNR de Chartreuse jouxtant l'agglomération grenobloise, peut paraître surprenant. Dès lors, les modes de fréquentation de la station évoluent vers des séjours plus fréquents mais moins longs afin de profiter des activités de loisirs offertes.

\section{L'immobilier de loisirs aux Sept-Laux}

Pour de nombreuses raisons, la station des Sept-Laux a connu un démarrage difficile. Tout en s'inspirant fortement du modèle de la station de troisième génération, elle reste en marge du Plan neige. Non seulement il ne s'agit pas d'une station savoyarde, mais surtout elle est implantée à proximité d'un centre urbain important et à une altitude relativement faible comparativement aux canons du modèle. Ces écarts avec la doctrine, ainsi que l'intervention de Laurent Chappis (architecte-urbaniste à l'origine de la station de Courchevel, station qui fut également celle de la rupture avec Maurice Michaud, ingénieur des Ponts et Chaussées, promoteur du Plan neige), vont freiner la concrétisation du projet de station.

La question immobilière constitue un enjeu central des débats. Laurent
Chappis propose, en effet, une conception en stade de neige qui va à l'encontre des mécanismes du modèle canonique (les bénéfices de la promotion immobilière permettant théoriquement de financer les investissements dans les remontées mécaniques). Dès lors, la station s'engage sur la voie d'un compromis peu convaincant : le refus de la conception en stade de neige débouche sur un nombre de lits trop limité pour constituer une véritable station de séjour, tout en bloquant le développement du tourisme en contrebas. En réalité, cette station, qui ne satisfait complètement aucune des thèses en présence, voit le jour surtout grâce à une forte volonté politique locale. Cette dernière est bien illustrée par la constitution d'un syndicat intercommunal dès février 1962 et se trouve incarnée par Aimé Paquet, député et conseiller général de l'Isère.

Mais cet ancrage local implique également des contraintes particulières. En effet, face au choix d'un promoteur toulousain, un groupement d'entrepreneurs locaux du BTP, Gamma 7, va jouer de sa capacité à faire pression pour se voir confier la réalisation des hébergements touristiques. Néanmoins, ce groupement va vite montrer son manque d'expérience et de savoirfaire dans la mise en œuvre d'un projet de station, notamment par son incapacité à assurer la vente des appartements construits. Il faudra attendre 1986 pour que s'achève un nouveau programme immobilier conduit par le promoteur Merlin, mais le boom de l'or blanc a d'ores et déjà marqué le pas. Après ce démarrage difficile, les péripéties rencontrées par la station des SeptLaux vont en effet se multiplier au fil des différentes crises financières. Elles conduiront notamment à l'intervention de la ville de Grenoble en 1985 et au retrait du département en 1992, à la suite de quoi un plan de redressement économique de la station sera mis en œuvre. Cette démarche va être à la base d'une réorientation globale de la stratégie de développement de la station qui se concentre sur son propre fonctionnement, affirmant sa vocation à produire des loisirs de proximité.

\section{Regards croisés}

sur le développement des loisirs

Dans le domaine des loisirs, la station des Sept-Laux affirme sa propre rationalité, héritée de sa trajectoire de développement, qui fait office de modèle de référence dans l'univers des stations périurbaines, notamment pour Saint-Pierre-deChartreuse. Le chiffre d'affaires lié aux clientèles de proximité est particulièrement marquant dans l'évolution parallèle de ces stations : leurs exploitants respectifs estiment ainsi cette part entre 75 et $85 \%$. La clientèle de proximité représente alors une véritable manne qu'il s'agit de séduire et d'attirer autant que faire ce peut. Néanmoins, ces clientèles ont un profil spécifique, centré sur la consommation du ski, raison première de leur venue en 
station ; elles sont particulièrement réactives aux conditions de pratique. L'enneigement constitue alors une des premières variables jouant sur la fréquentation. L'installation de webcam renseignant le client potentiel en temps réel fait ainsi partie des moyens de commercialisation du domaine skiable particulièrement répandus et auxquels les deux stations périurbaines souscrivent. Elles se distinguent néanmoins par leurs investissements dans les équipements d'enneigement artificiel assurant le retour "skis aux pieds" au bas des pistes. Les Sept-Laux a consenti un important effort financier dans ce sens, alors que SaintPierre-de-Chartreuse n'a pas réussi à dégager de tels moyens.

Un segment de clientèle est particulièrement courtisé, celui des jeunes et étudiants. Dans ce sens, les Sept-Laux développe une véritable stratégie de communication dédiée à cette cible, notamment à travers la présence de la station dans la communauté internet (en particulier par le biais de forums de discussion spécialisés, comme skipass.com). Dans la même logique, les deux stations ont mis en place une tarification adaptée à une fréquentation de proximité, même si cet outil peut se révéler attractif au-delà du seul segment “jeunes". Une politique tarifaire agressive à destination de la cible des étudiants vise à lisser l'exploitation du domaine sur la semaine. Saint-Pierre-de-Chartreuse propose, par exemple, un forfait "bradé" pour les étudiants, entre le lundi et le vendredi. La station vend également un forfait "petite journée" à partir de 11 heures, pour répondre à la décision spontanée de monter en station des clientèles proches. Les Sept-Laux joue également cette carte de la flexibilité à différents niveaux : d'un côté avec la mise en place du système Temposki qui permet de créditer sur un compte les heures de ski non consommées sur un forfait et, de l'autre, en jouant sur le prix de vente des forfaits en fonction de la proportion du domaine skiable ouvert.

L'objectif n'est donc plus d'attirer des touristes en séjour, mais de maximiser l'exploitation des remontées mécaniques en étalant au maximum leur fréquentation afin de réduire les périodes d'exploitation déficitaires. En cohérence avec ces objectifs, des aménagements de loisirs se multiplient sur le domaine skiable sur le thème des nouvelles glisses, thème particulièrement cher aux Sept-Laux et qui se développe aussi en Chartreuse.

\section{CONCLUSION}

\section{DÉTERRITORIALISATION ET}

RETERRITORIALISATION :

\section{VERS UNE NOUVELLE ÉCHELLE DES DESTINATIONS}

Dans le modèle du Plan neige, la rentabilité des remontées mécaniques repose avant tout sur la clientèle de séjour, supposée captive et entièrement tournée vers le ski. En ce sens, l'équilibre entre les hébergements et le domaine skiable (le débit des remontées mécaniques) constitue une garantie réciproque de cohérence : le remplissage des premiers assure que les équipements ne tournent pas à vide, les remontées de leur côté mettant en valeur le site et favorisant la location des hébergements marchands. Cette logique est particulièrement importante dans le cadre d'une station village comme Saint-Pierrede-Chartreuse, l'attractivité du domaine skiable gageant de la diffusion locale du développement.

La question se pose donc de l'évolution du rapport de réciprocité entre ces deux tendances. D'une part, la diminution des hébergements marchands réduit non seulement la capacité du territoire support de la station à contribuer au fonctionnement des remontées mécaniques, mais également la capacité du territoire à profiter de l'apport de revenus touristiques. D'autre part, le gestionnaire du domaine affirme sa propre rationalité économique en se développant comme un centre de profit au sein du territoire, mais dont les effets d'entraînement sont d'autant plus limités qu'il oriente son offre vers une clientèle focalisée sur une pratique de loisirs.

Dans le cas des Sept-Laux, la situation apparaît sensiblement différente dans la mesure où, dès l'origine, la question immobilière s'est révélée très problématique et le développement du tourisme relativement distinct de celui des communes supports. Par ailleurs, le 
phénomène de périurbanisation a surtout touché les villages à plus faible altitude que la station ellemême, dont l'immobilier paraît peu propice à la résidence permanente. Eu égard au démarrage de la station et à la réalisation incomplète des premiers programmes immobiliers, le site aménagé dispose aujourd'hui de marges de manœuvre sur le plan foncier. Le nombre de ses logements ne constituant pas un facteur critique pour le bon fonctionnement des SeptLaux, cette marge de manœuvre peut être exploitée dans une double optique de revalorisation de l'image de la station et d'usage futur. En effet, un projet de construction de logements neufs, Les Granges des Sept-Laux, a été réalisé sur le site de Prapoutel sur des critères de surfaces très différents de ceux retenus pour les studios Merlin. Ce projet s'inscrit très clairement dans la volonté de "positiver" l'image et de créer du désir dans une "tentative" de territorialisation sur le mode néo-régional, que d'aucuns qualifient de style "tyrolosavoyard", contribuant à la diffusion d'une image galvaudée de la montagne (sous-bassements en pierre apparente, bardeaux de bois, balcons savoyards, etc.). Cette démarche n'est d'ailleurs pas sans rappeler l'opération de rénovation des immeubles plus anciens qui s'est finalement concentrée sur le ravalement des façades. Par ailleurs, les critères de confort revus à la hausse laissent envisager, comme le relatent les entretiens auprès des décideurs locaux, une utilisation des bâtiments au-delà du tourisme. En cas de changement climatique, d'extension du phénomène périurbain qui déborde d'ores et déjà sur la station pour les individus dans les situations de précarité les plus marquées, ou tout simplement d'arrêt de l'activité touristique, ces logements pourraient, plus facilement que les studios cabines, répondre à une demande de résidence permanente.

Si les deux tendances présentées in fine apparaissent de prime abord radicalement différentes, force est néanmoins de constater qu'elles convergent pour donner une nouvelle forme de territorialisation des stations. Dans sa conception, l'ancrage des stations est avant tout un ancrage productif, le territoire support fournissant les matières premières à exploiter et bénéficiant en retour des fruits de leur exploitation. Aujourd'hui, les stations semblent plutôt se diriger vers un "ancrage produit", comme une manière de distinguer leur offre de ski dans le jeu de la concurrence. Ce faisant, le territoire d'ancrage change d'échelle.

Conçues sur la base d'une rationalité fonctionnelle des processus productifs, les stations se sont à l'origine centrées sur elles-mêmes, avec comme périmètre de référence de gestion celui de la commune. Actuellement, ce périmètre éclate. Dans le cas des Sept-Laux, cet éclatement se construit en continuité avec l'agglomération, comme un lieu de pur loisir périurbain permettant un accès ludique au milieu montagnard. La prégnance de cet ailleurs compensatoire est particulièrement visible dans la capacité de la station à attirer une clientèle lointaine de passage dans l'agglomération. Pour autant, l'agglomération n'est pas absente de la logique chartroussine. Les relations sont toutefois d'une autre nature et la station de Saint-Pierre-deChartreuse tend plutôt à retrouver une nouvelle cohérence dans la structuration d'une offre de loisirs plus globale au sein du PNR de Chartreuse. Celui-ci, en effet, développe une filière neige raisonnée à l'échelle du parc ; le slogan utilisé n'hésitant pas à mettre en avant l'altérité de l'ensemble au regard du monde urbain en contrebas : "si proche et tellement différent".

Le paradoxe réside finalement dans la convergence des offres de tourisme de chacune des deux stations étudiées et dans le spectre de la banalisation qui plane sur leur avenir : d'un côté, la construction d'hébergements "de caractère", de l'autre le développement de prestations de loisirs parfois en décalage avec les réalités locales (par exemple, l'aménagement d'un parc aventure forestier générique (banal) alors que l'exploitation forestière tient une place centrale dans l'histoire de la commune). Dans ce sens, nos cas d'étude se rapprochent inexorablement de parcs à thème "montagne" dans lesquels 
des offres comparables émergent à travers la multiplication des prestations de loisirs (VTT de descente, nouvelles glisses, handisport, etc.) dans un écrin dont la typicité relève souvent de l'affichage. Outre la fréquentation garantie par la proximité urbaine, ce mode de développement peut-il être viable à long terme ou bien relève-t-il plutôt d'un effet de mode? La diffusion de ce type de stratégie pourrait-il supporter la concurrence d'une offre de type resort ( $c f$. le "village" d'Arc 1950 implanté par la société Intrawest)?

(I) Des informations complémentaires aux deux cas d'étude présentés pourront être recherchées dans le rapport de recherche complet rédigé par les auteurs (François et Billet, 2006) ; ce travail ayant été réalisé au sein du programme "Nouvelles pratiques touristiques en zone de montagne : vers un renouvellement des pratiques de gestion foncière ?" coordonné par Emmanuelle Marcelpoil (Cemagref de Grenoble - UR DTM) et financé par la direction du Tourisme.

(2) Enquête réalisée durant la saison 2005 2006 sur des périodes de week-end et de semaine pendant et hors vacances scolaires auprès de 386 personnes. Pour plus de précisions et la lecture des résultats détaillés, le lecteur peut se reporter aux travaux de thèse de l'auteur (François, 2007).

\section{RÉFÉRENCES BIBLIOGRAPHIQUES}

Vincent BRIQUEL, "L'avancée de la périurbanisation dans les Alpes du Nord françaises et ses liens avec la croissance récente de la population", Revue de géographie alpine, I, 2001 .

Christopher BRYANT, "La place des espaces ruraux périurbains et de l'environnement dans le développement local', Symposium international : Territoires et enjeux du développement régional, 9-1 I mars, Lyon, 2005.

Pascal Cuvelier, L'Économie des pratiques touristiques, thèse de doctorat, Sciences économiques, sous la direction de Jean GADREY, université des sciences et technologies de Lille, 1997.

Laurent DAVEZIES et Patricia LejouX, "Un train peut en cacher un autre. Derrière l'économie productive, attention à l'économie présentielle", XXXIXe colloque de I'ASRDLF : concentration et ségrégation, dynamiques et inscriptions territoriales, Lyon, I-3 septembre, 2003.

Hugues FRANÇOIS, De la station ressource pour le territoire au territoire ressource pour la station. Le cas des stations de moyenne montagne périurbaines de Grenoble, thèse de doctorat en sciences du territoire, sous la direction de Bernard Pecqueur et Emmanuelle Marcelpoil, université Joseph Fourier, Cemagref Grenoble, 2007. Hugues FrançoIs et Sylvain BILLET, Enjeux touristique, foncier et immobilier pour les stations de moyenne montagne : la nouvelle donne périurbaine, rapport pour la direction du Tourisme, Cemagref Grenoble, 2006.

A.-L. GuILlAUd-LAUZANNE, Les conflits de pratiques foncières entre périurbanisation et parc naturel régional. Le cas du PNR de Chartreuse, mémoire de DEA, Géographie, sous la direction de Sylvie Duvillard, Institut de géographie alpine (IGA), 2004.

Rémy KNAFOU et Philippe VIOLIER, "Les mondes urbains du tourisme",

EspacesTemps.net, n²3-06-2005 [http://espacestemps.net/document l446.html, 2005].

Olivier LazZAROtTI, "Les loisirs périurbains", Espaces, I27, 1994.

Olivier LAZZAROTTI, "Tourisme et espace périurbain : dépasser les contradictions?", dans Driss Boumeggoutı (dir.), Le Périurbain : une zone touristique d'avenir, Toulouse, univ. du Mirail, 2005 ( $n^{\circ}$ spécial revue Tourisme).

Isabelle Mauz, Denis Malavieille, Marie-Christine Micheels et al., Enjeux socio-économiques et capacité d'adaptation des stations de moyenne montagne - Analyse d'un échantillon de trois stations - 2. Saint-Pierre-de-Chartreuse, rapport pour Afit-Seatm, Cemagref - UR DTM, 1996.

Jacques Perret, Le développement touristique local. Les stations de sport d'hiver, Économie du développement, sous la direction de Claude COURLET, Université Pierre-Mendès France - Grenoble 2, 1992.

Hubert SCHWITZ et Khalid NADVI, "Clustering and industrialization : introduction", World Development, vol. 27, n 9, 1999. 